\title{
CAM Modalities Can Stimulate Advances in Theoretical Biology
}

\author{
Alex Hankey \\ Hethe House, Cowden, Kent TN8 7DZ, UK
}

\begin{abstract}
Most complementary medicine is distinguished by not being supported by underlying theory accepted by Western science. However, for those who accept their validity, complementary and alternative medicine (CAM) modalities offer clues to understanding physiology and medicine more deeply. Ayurveda and vibrational medicine are stimulating new approaches to biological regulation. The new biophysics can be integrated to yield a single consistent theory, which may well underly much of CAM-a true 'physics of physick'. The resulting theory seems to be a new, fundamental theory of health and etiology. It suggests that many CAM approaches to health care are scientifically in advance of those based on current Western biology. Such theories may well constitute the next steps in our scientific understanding of biology itself. If successfully developed, these ideas could result in a major paradigm shift in both biology and medicine, which will benefit all interested parties-consumers, health professionals, scientists, institutions and governments.
\end{abstract}

\section{Introduction}

In the past decades, the public have turned to therapies of complementary and alternative medicine (CAM) on a scale that ministries of health were unprepared for, and which is driving the present revolution in patterns of health care (1-6).

One reason is the increased incidence of chronic disease, and the natural concern of many people to leave no stone unturned in their search for an end to the suffering of themselves, or their nearest and dearest, particularly if someone they trust has found relief. It is commonplace that the increase in mean ages of their populations is driving the increase in chronic disease in developed countries (7). As baby boomers come up to retirement, a crisis in public health looms that will stretch the budgets of all economies.

Currently, orthodox biomedicine has no power to cure these diseases. Indeed, that is the only reason orthodox medicine lumps such illnesses together in the category it terms 'chronic', introducing the terms 'chronic disease care' (8) and 'chronic disease management' (9) to indicate that the best it can offer is being done for them.

On past experience, conventional medicine will only discover cures for chronic diseases slowly. It is therefore imperative for all concerned that other avenues of approach to the problem are explored. Wherever CAM modalities can be shown to offer cheaper or more problem-free alternatives to conventional practice in managing chronic disease, they will potentially provide welcome relief for Ministries of Health and their Exchequers alike. If such CAM therapies also offer fewer side effects than drug therapies, they will be welcomed by consumers. Any CAM modalities that were shown to provide reliable cure for a presently incurable chronic pathology should be doubly welcomed.

One of the problems that faces governments in providing funding for CAM therapies, however, is the continued lack of understanding of what they can offer and how they work. On the one hand, the evidence base of complementary medicine is increasing, an important factor this journal has been founded to encourage (10). On the other hand, there is an almost complete vacuum of theoretical understanding of complementary medicine. This presents a block to those who make decisions on whether specific therapies merit public funding based partly on scientific models of how they function-models that become increasingly sophisticated, as science progresses. For this reason, the need for developing scientific theories of how CAM modalities work is of significance in order to justify using them on a wider scale $(11,12)$. Indeed, Cooper $(10)$ specifically states that every concept within CAM should have its biology, a perspective which this papers upholds, and the fulfilment of which could greatly increase the acceptance of CAM.

The significance of this is illustrated by the history of Lord Walton's Report on CAM to the UK Government in 2000 (13). After the preferred CAM modalities of homeopathy, osteopathy, chiropractic, acupuncture and herbal medicine in category I, others were classified in four lower categories, with the least 
comprehensible being relegated to the lowest, IIIb, despite the fact that homeopathy was as incomprehensible as any-though this may no longer be the case. Of particular concern was the fact that traditional systems of medicine such as Ayurveda and Traditional Chinese Medicine received the most unjust treatment (14), provoking protest from the governments of their countries of origin. This was entirely due to their theoretical bases being incomprehensible to the subcommittee, and their literatures being in tongues equally unknown.

It is the experience of this author, shared by colleagues trained in the practice of traditional systems of medicine, that such systems have self-consistent theoretical underpinnings. They have been practiced for millennia by intelligent members of supremely intelligent civilizations. To dismiss them without careful investigation is to risk losing much of potential value from their vast experience and long history of development.

It has been the working hypothesis of this author that such traditional systems, and indeed other modalities of CAM, use levels of physiological functioning that science has yet to recognize (11) let alone understand. This approach has resulted in three radical theories, one on Ayurveda's Tridosha system of classification of physiological function and regulation (15-17), one on a theory of physiological regulation (18), which results in a theory of vibrational medicine (19) including homeopathy (20), and one (21) which draws on the other two to begin to explain one of Scott-Morley's many advances in the practice of Voll's system of electroacupuncture (22).

One compelling aspect of these theories is their compatibllity. They yield insights into previously unsuspected aspects of physiological function which corroborate each other; there appears to be a single overall theory. If the theory can be extended to include further aspects of CAM, based on the insights it already provides, each step of progress should yield new insights into physiological function, and provide deeper understanding of CAM as a whole. The next section briefly reviews the new theories, which are then presented in more detail.

\section{The Biophysics of Regulation and Health}

Phenomenologically, any theory of health and deviation from it requires a theory of biological regulation-for two reasons. First, for an organism to function efficiently, its processes must be regulated. To compete more effectively, it must be more efficient. Hence, all biological processes should be regulated. Concepts of regulation must be central to understanding all biology, an idea confirmed by the central position of homeostasis and homeodynamics in modern physiology and medicine. Secondly, the way organisms respond to challenges in internal and external environments depends on their natural regulatory processes. Normal adaptive functioning is only transformed into a maladaptive response when regulatory systems fail under the pressure of over-stressful challenges. Maladaptive responses are the precursors of pathological malfunction (23). It follows that theories of regulation and their failure under stress must be central to biophysical theories of the transformation of health into disease.
This is amply confirmed by much work now being published on cancer and regulatory processes involved in oncogenesis (24-26). Such work is being carried out on the microscopic level, from a reductionist perspective, e.g. the effects of failure of genetic regulation on single enzyme activity is often the focus for such studies. However, the microscopic, reductionist approach fails for the study of CAM. Systems of complementary medicine tend to act on a more holistic level, and require more holistic approaches to describing physiological function for theories of their action to be constructed. A fundamental reason why complex organisms exhibit holistic functioning lies in the fact that regulation processes must themselves be regulated. This leads to an integrated hierarchy of regulation processes of an holistic nature. Assuming that regulation processes are necessarily independent of each other-the reductionist perspective-is therefore a fundamental error when considering the regulation of complex biological systems.

Hyland (27) specifically suggests that systems theory and the physics of complexity must be necessary to describe processes in holistic medicine. Holistic aspects of systems are necessary to describe similar properties of holistic medicine. Ordinary physics and chemistry cannot do so. Milgrom (28-31), in an equally radical approach, has used properties of quantum systems to model several aspects of homeopathy. Similarly, considering the problem of how homeopathic remedies interact with the physiology, Torres (32) has shown that instabilities in the physics of networks can model aspects of how they do so. $\mathrm{He}$ concludes that such critical instabilities, as they are known, may provide a starting point for theories of homeopathy.

Such work has long been anticipated. Bodeker (33) points out that concepts in traditional systems of medicine are closer to physics than to chemistry. New biophysics may make it possible to understand such systems. There have been corresponding developments from the biological side. Frohlich (34) found concrete evidence for coherent behaviour in biological systems, implying that additional levels of orderliness are unexpectedly present. Such additional orderliness can result in more integrated function, and be the basis of truly holistic patterning. Describing it will require the physics of complexity. Smith shows there is evidence for quantum processes, normally found only on the atomic level, operating on a macroscopic scale (35). He has called for the development of a 'physics of physick' (36).

All the above inputs $(23,27-36)$ complement each other. Taken together, they identify characteristics required of theories of CAM: they should be holistic theories of regulation, built around systems theory and the physics of complexity, involving quantum theory and critical instabilities to yield macroscopic wave functions and corresponding patterns of orderliness and coherence. Remarkably, the theories of biophysical regulation presented below $(15-18,20,21)$ embody all these properties, and may be integrated into a single coherent theory-a first step towards a biophysics of health.

The following section summarizes a theory of Ayurveda's Tridosha $(15,16)$ utilizing systems theory $(27)$ to identify the chief functions requiring regulation. The subsequent section shows how the theory of critical regulation (18) at critical 
instabilities (32) combines large-scale correlations (34) and the physics of complexity (27) to produce macroscopic quantum wave function mixtures (35), which can model the action of vibrational medicines (19) and homeopathy (20,28-31). The final section integrates these two theories to model some of Scott-Morley's discoveries (21). This shows that the new biophysics applies to traditional systems' description of deviation from health $(33,37)$ qualifying it as a 'physics of physick' $(36)$.

\section{Ayurveda's Tridosha: Gross Regulation of Organisms}

Ayurveda, the system of medicine from the Vedic civilization of ancient India, analyses health in terms of 'displacement from perfect balance' of three principles named doshas, and their 15 subdoshas (37). It lists several levels of dosha imbalance occurring before pathology manifests, and so classifies states of health as well as disease. Correcting these, Ayurveda restores health, as well as eliminating pathology (38). The overall concept is called Tridosha.

By analysing the functions ascribed to the doshas, it has proved possible to develop a scientific theory for them in terms of elementary systems theory, which identifies three necessary functions for any open system, input/output, throughput or turnover and storage or structure (15). Any open system communicates with the external world, hence input and output occur (Fig. 1a). In any non-trivial system, there must be processing between input to output, so 'throughput' or turnover must also take place (Fig. 1b). If the latter is not to be a mere conveyor belt, there must be some process where items in throughput are put on hold for future use, i.e. storage (Fig. 1c). Hence some form of all three exist in any non-trivial system.

Mandatory organism efficiency, discussed above, requires that these be regulated. By comparing the doshas' known functions with these three, the three doshas, Vata, Pitta and Kapha can be identified with the necessary control functions regulating input/output, throughput or turnover, and storage or structure, respectively. For example, Vata is responsible (among other things) for nutrient and fluid uptake in the colon, its peristaltic action and also kidney function. It tends to involve processes requiring membrane transport, such as nerve function. Pitta regulates digestion and all processes of energy regulation and balance, e.g. skin pigmentation. Kapha is responsible for structure, cohesion and lubrication, the relationship of which to storage is based on a common biochemistry of lipids and polysaccharides (16).

In single cells, the same functions are governed by similar biochemical processes: input/output, Vata, membrane transport; energy turnover, Pitta, metabolism; and storage, Kapha, lipids in the cell membrane and carbohydrates in the cell wall. Similarity of biochemical function makes it possible to trace a continuity of Tridosha function thoughout biological evolution. The only possible conclusion to be drawn is that the three doshas are invariants of evolution. As such they are universal principles of biological regulation, present in all organisms. Since they depend on systems theory, rather than details of (a) INPUT / OUTPUT

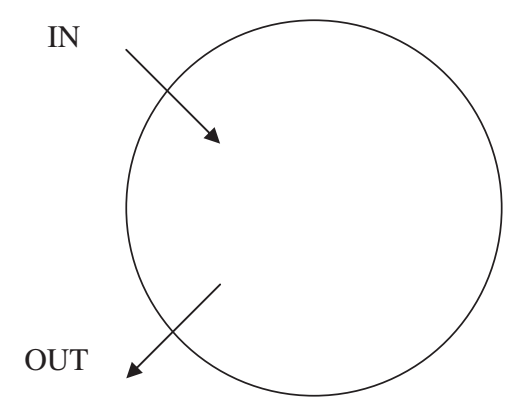

(b)

THRUPUT / TURNOVER

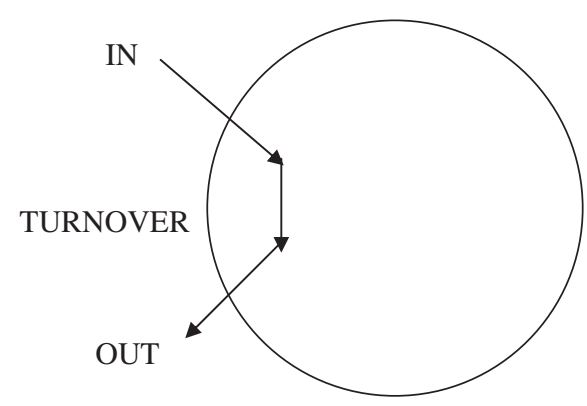

(c)

STORAGE

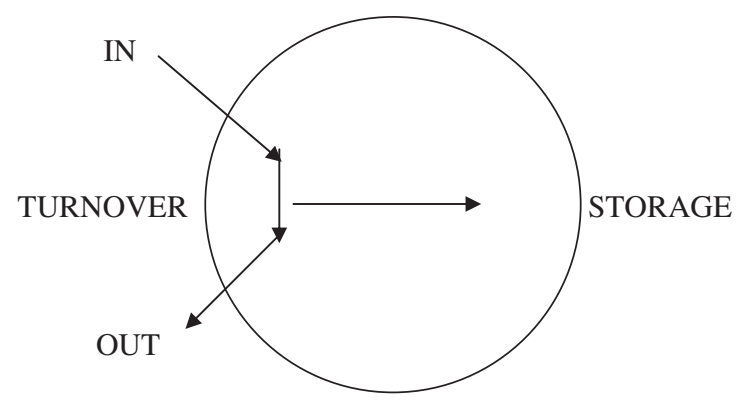

Figure 1. (a) Input and output processes are necessary for any system to be an 'open system'. (b) Turnover connecting input and output processes is necessary in any non-trivial open system in which what goes in does not simply come back out. (c) Storage, as a means of bringing materials on and off the turnover 'production line', is a second essential process in any non-trivial open system. Then useful materials can be used at a later time, or components can be combined in an order different from that in which they entered.

biochemical structures, they are more fundamental than DNA and the genetic code, the apparently arbitrary choices in which make them specific to life on earth.

The systems approach has been justified by considering the pathway of fatty acid metabolism using acyl coenzyme A, occurring in all cells in all life forms (16). Its universality implies that it plays a role of exceptional significance. Only the systems approach can adequately explain this. It is the regulated metabolic pathway connecting Pitta and Kapha functions: metabolism (or energy turnover and negentropy production) and the storage of energy-rich molecules in the cell 
membrane and cell wall. Only its fundamental role in the strategic design of life processes can explain its continuing presence in all cells, when mutations creating alternative pathways and functions arise so frequently in biological history (16).

That dosha functions remain in some sense unchanged and invariant during evolution, like, for example, the genetic code, is supported further by considering the principal locations of the doshas in the human body: Kapha in the head and chest, Pitta in the middle abdomen and Vata in the lower abdomen. Their functions reveal the gastric canal to be the key to understanding their separation: Kapha provides mucous lubricants to smooth the flow of chyme and protect the gut walls from potentially damaging materials ingested along with food, and must come first. Pitta generates digestive enzymes, necessarily preceding the absorption and elimination functions of Vata, which must come last. This means that the principal locations of the doshas must have separated in this precise order with the development of the gastric canal, and have remained the same-invariant-ever since (17).

Further consideration shows that each dosha corresponds to one of the principle definitions of life itself:

(i) regulation of input/output, or Vata dosha, governs homeostasis, the ability of living organisms to regulate their own internal environment;

(ii) regulation of metabolism, or Pitta dosha, enables the living organism to function far from equilibrium, and so generate the negentropy needed to maintain its integrity of structure, including its internal environment;

(iii) regulation of storage and structure, or Kapha dosha, is connected to an organism's ability to maintain its characteristic appearance or form-historically, the first and most common definition of how to identify and characterize different forms of life.

These modern biological explanations for traditional properties ascribed to the doshas show that, as Ayurveda's traditions maintain, they are indeed fundamental properties of all living organisms, not just human physiology. This demonstrates the real significance of dosha. It is a foundational concept of life. Tridosha constitutes the most compact, holistic account of organism regulation ever proposed, integrating all three of its fundamental aspects. Far from describing something esoteric or culturally specific, ancient Ayurvedic texts identify key properties of living organisms, more precisely and economically than modern medical text books, and in a way that is more relevent to the disease process, since their maladaptative functioning is directly involved in, and fundamental to, all etiology.

In light of these ideas, empirical confirmations of the concept of dosha assume fundamental importance. They are of two kinds. First, statistical analysis of questionnaires used to measure doshas confirms the validity of the concept (39), in a similar way that concepts in psychology of individual differences are validated by similar statistical analyses. Secondly, the dominance of one or more doshas in individual functioning of the physiology, known in Ayurveda as the Prakriti of the individual, has been shown to have genomic correlates (40)-and for good reason, dosha invariance throughout evolution requires the functions and their variations to be genome based (17).

\section{Genomes and Feedback Singularities}

The doshas have a deeper significance in Ayurveda than the description of physiological processes. They also describe the dynamics of organismic health. When a maladaptive reaction takes place, one or more of the doshas or their subdoshas are said to 'go out of balance' $(15,37)$, a process which can be detected by changes in their qualities in the pulse (38). Descriptions of how the doshas go out of balance involve successive processes of different character, and sound like different kinds of phase, in a phase diagram, with phase transitions between each process (15).

To understand why and how phase transitions arise in this context, consider regulation processes in more detail. Regulation involves switching between two states, in one of which the regulated process is switched on, and in the other, it is switched off. If such a switching process results in a change in concentration of some chemical species, it is analogous to a phase transition for the following reason. In non-equilibrium thermodynamics of complex chemical systems such as occur in living cells, a discontinuous change in the concentration of even a single chemical has the same mathematical properties as when that chemical is the only one present. A discontinuous change in the concentration of a single chemical, such as the transition from a liquid to a gas, results in a visible change of phase. Even when the manifest nature of the phase change is obscured by many other chemicals being present, from a theoretical perspective, a phase transition has occurred, albeit one that is not so manifestly obvious, simply because the mathematics describing the discontinuous process are the same.

In their book Order Out of Chaos, Prigogine and Stengers (41) take this a stage further, and point out that feedback required for regulation and control in biological systems results in the same instabilities that Prigogine had previously studied in far-from-equilibrium thermodynamic systems. Such instabilities occur at the boundaries of phase transition regions; they may be points at the end of a line of phase transitions representing on/off switching processes. This means that switching processes and regulation always have such instabilities associated with them.

A crucial point in connecting this to complementary medicine is that such singularities can be mathematical attractors in the time evolution of a system's dynamics. In this case, left to itself over a sufficient time period, the system will naturally centre itself on the instability. The instability with its instability fluctuations will dominate the system, a condition termed 'critical regulation', with many significant consequences (18).

One is that a form of self-organized criticality (SOC) results. SOC is very important in the physics of complexity, for it simplifies system response to external stimuli. In contrast to ordinary SOC, however, this is not an 'emergent' property of the system, but inherent, since the feedback generating the singularity is necessary for cellular functioning. The existence 
of SOC as a general property of biological systems can explain the observations noted by Torres (32) and Frolich (34) since both directly imply critical behavior. SOC means that living systems possess large-scale coherence, a property of instability fluctuations at their critical points. Secondly, the elements that 'fluctuate' are quantum expectation values of biochemicals involved in regulation. These couple to genome expression and may mean that activity at active sites 'fluctuates' in a similar quantum sense. On a quantum level, this has the advantage of allowing fine tuning of the system to take place, permitting much more precise (and thus more efficient) genetic expression in response to given levels of demand (18).

For example, in genomic regulation, a regulatory protein binding to the DNA may prevent a gene from being read, and therefore switch it 'off'. When the protein is itself switched off, the site it represses is switched on. In eukaryotic cells of multicellular organisms, gene repression is the fundamental strategy of regulation, required in order to prevent too many genes being expressed in differentiated cells and decreasing cell efficiency.

Instability fluctuations smooth regulation processes and prevent systems falling into unwanted phases. If a regulatory system departs from its critical instability point because of 'pressures of operation', i.e. the 'stress of life', it becomes susceptible to getting stuck in a metastable state of some phase, in which a required function may be switched off, giving the phase a pathological property.

If the instability point is an 'attractor', resting will allow the system to return there, restoring power to the critical fluctuations, function to the regulated system, and removing the pathology. This is reminiscent of naturopathy, where rest restores health by allowing the life force to regain its required energy level. In this case:

(i) the life force is equated with quantized instability fluctuations; these are strong close to the instability, but become weaker going away from it;

(ii) pathology arises because the system is driven away from its natural resting place, and becomes stuck in a metastable state in the wrong phase of the phase diagram near the instability;

(iii) i.e. an imbalance has arisen in the regulatory system, the doshas;

(iv) rest allows the system to return towards the dynamic attractor;

(v) close to the instability, the fluctuations are stronger; so that

(vi) their restored power re-exerts regulatory control;

(vii) the system moves out of its metastable state in the pathological phase;

(viii) functionality returns to the regulated function; and

(ix) health is restored.

This presents a theory of the life force in terms of dynamics of regulation of cellular function, at cytoplasm and genomic levels, avoiding all the difficulties that led to its being rejected in the middle of the last century. Furthermore, since it explicitly relates how doshas go out of balance to a phase transition process, it links this section, concerned with the mathematical properties of regulation, to the previous one, concerned with the systems analysis of organism function. The theory can also be applied to the 'vibrations' of vibrational medicine (19), such as homeopathic medicines: they are quantum fluctuations, with similar power to stimulate phase transitions in living systems stuck in a metastable state $(18,20)$. The addition of quantum fluctuations in the form of a vibrational medicine (of whatever kind) adds power to the naturally occurring life force, as many phenomenological accounts of their actions state, and naturally restores a system to a healthier place in the phase diagram.

One key idea in chronic disease is that the life force has become weak. The only way to restore its power is to strengthen it, i.e. with the quantum fluctuations of which it is constituted. Mere drugs acting at a chemical level will never achieve this. Vibrational medicine can, however, if the correct fluctuations are administered. Those of a toxin coupling chemically to the disabled function (i.e. the function it poisons) will do so (18). This forms the first detailed scientific theory of the homeopathic principle, and is probably the most powerful result established for the theory so far.

\section{Ayurveda and Energy Medicine}

The implications of the last two sections are far reaching. On the one hand, the majority of Western-trained doctors who are also practitioners of Ayurveda concur with Eastern-trained traditional practitioners that all patients presenting pathological states have easily detectable imbalances in their doshas (37). On the other hand, practitioners of various forms of energy medicine, including vibrational medicine, find that pathological states of the physiology can be restored to healthy states by means of appropriately prescribed energy medicines.

Furthermore, the two systems also agree in a second way: the precise imbalances are patient specific. In Ayurveda, imbalances arise as a result of stressors acting on the patient's prakriti and life experience, and require treatment as much as the presenting symptom. The same disease according to the classification of Western pathology can be the result of a different sequence of imbalances in the patient according to Ayurvedic etiology.

Imbalances are person centered and patient specific, in contrast to diseases which are, according to Western classification, presenting symptom specific. This is in fundamental agreement with all systems of energy medicine. Acupuncture (and its developments) detects imbalances in meridian energies and corrects these as much as addressing the presenting symptoms. Homeopathy as developed by Hahnemann is concerned with life history and with the similarities of subtle levels of symptom, not necessarily related to the presenting symptom, to those of specific toxins. Again, a much wider symptomatology is taken into account and the treatment becomes patient specific, rather than presenting symptom (and therefore disease) specific.

These more subtle symptoms can all be understood as being caused by subtle imbalances in the regulatory system. It is natural, therefore, to hypothesize that naturopathic medicine, 
Ayurveda, Traditional Chinese Medicine and acupuncture, and other forms of energy medicine including vibrational medicine and homeopathy, are all working on the same basis: correct the problems in the regulatory system of the patient. Restore the regulatory system to its state of healthy functioning in which the life force maintains its 'state of balance' and is able to provide full regulation.

The real advantage of the new biophysics of cell regulation is now apparent. It presents a picture of biochemical regulation based on well accepted physical chemistry which can unify our understanding of separate disciplines of CAM. Having established that patient-specific etiology, imbalances in dosha and subdosha functioning, can be accounted for in terms of phase transitions in a phase diagram (15), the new biophysics begins to explain how, in providing cures, naturopathic medicine, energy medicine and vibrational medicine including homeopathy can reverse the phase transitions and restore balance. A genuine 'physics of physick' is beginning to emerge as a description of etiology in terms of physical chemistry incorporating the physics of complexity.

In support of this, it is already recognized that homeopathy and energy medicine are connected to Ayurveda. Specific miasms are known to correspond to specific dosha imbalances according to one practitioner trained in both homeopathy and Ayurveda who finds the relationship helpful in his prescribing, e.g. life style modifications for specific miasms based on their corresponding Ayurvedic dosha properties (Dr Peter Hudson, personal communication). The presence of a miasm implies that a phase transition has taken place in the physiology, recognizable as a specific transition (imbalance) in dosha functioning.

Similarly, Scott-Morley has discovered three deeper layers of pathology encoded within the acumeridian system, accessible by the bioresonance test combined with feedback (22). His reports that these can be systematically eliminated with energy medicines (electronic analogs of homeopathic remedies) can be scientifically modeled by the new theory (21). These observations would tend to indicate that the acumeridian system is also concerned with the biophysics of regulation, as would be expected from the analysis of the disease process given above. Electroacupuncture then provides quantitative measures of 'imbalance' in a regulatory system. This implies that the acumeridian system can be expected to be governed by analogous biophysics to that described above-Torres's concepts of critical points on networks (32) may come into their own, providing new forms of long-range coherence (34) and macroscopic quantum fields (35).

The richness and potential versatility of the new biophysics should now be apparent. Hopefully, this will stimulate interest in developing further applications. Whatever the theoretical interest, it is in potential applications to chronic disease that the value of the new insights may truly blossom. A theory has been provided for why the same chronic condition (according to biomedicine's classification) may correspond to different imbalances and classification according to disciplines of CAM. In certain cases, the correct discipline of CAM may be able to reverse the imbalances to the extent of providing a cure for specific patients with the chronic condition (42). That is not to say that a chronic condition becomes a curable Western pathology, but that certain identifiable sufferers (possibly a substantial proportion) could have their condition reversed and, in regaining health, cease to be a burden to insurance companies and the state.

\section{Conclusions}

As Selye proposed, pathology is initiated by a maladaptative reaction to a stressor (23). Maladaptation is a failure of a natural process of regulation or defense. Hence the reasons for pathology depend on specific stressors to which the patient has been exposed, combined with the failure of natural regulation processes and/or defense mechanisms.

Leaving aside the question of stressors, and focusing on the patient, the key to the scientific approach to etiology must lie in a theory of regulation. Tridosha in Ayurveda presents the holistic structure of regulation in any given organism, showing how to classify the regulatory systems that can go wrong, i.e. any failure of natural regulation processes must be an 'imbalance in the doshas'. Modern biophysical theories of organism regulation based on quantum theory and critical phenomena yield a selfconsistent theory of the life force and how its deformation results in the maladaptive reactions leading to pathological states.

These theories have the power to describe many unusual phenomena, including those identified by several different disciplines of CAM. Examples given here illustrate this idea for Tridosha, naturopathic medicine and vibrational medicine, promising to explain much of the physics behind physick.

The proposed biophysics of regulation may therefore be regarded as the beginings of a possible biology of complementary medicine, albeit one requiring a great deal of refinement and many extensions. It is greatly to be hoped that theories such as these will help secure the acceptance of CAM disciplines as valid in their own right within the framework of modern academic medicine.

There is an acute need for theory both to persuade the more conservative members of the scientific and medical communities that CAM disciplines have genuine substance and because, to increase the evidence basis for CAM, a set of testable theories is needed to guide experimental design (12). For example, it has been made clear here that prescribing vibrational medicines, or other forms of energy medicine, on the basis of presenting symptom alone, is likely to decrease the effectiveness of treatment. How much worse for the evaluation of CAM disciplines if biomedical education alone is used to guide experimental design and study protocols.

There should be no illusion that we are close a complete theory of all disciplines of CAM. Many CAM concepts still present scientific mysteries and must be approached with the same willingness to seek clues as to how the missing theories of their biophysics may be constructed. Take, for example, the reflexological maps familiar to every practitioner of Traditional Chinese Medicine. To the CAM community, their validity is undoubted, but no hint of their biophysical or physiological 
basis is as yet forthcoming. Similarly, the modus operandi of the nadi/acumeridian system, the way it coordinates biological activity, is the subject of interesting hypotheses, but no properly tested theory has yet emerged. Nor has any fundamental reason for its existence in both animals and plants been hypothesized.

Like doshas, reflexological maps and the nadi/acumeridian system are fundamental to animal physiology. Both these problems presented by CAM proclaim fundamental properties of life we have yet to understand and identify in scientific terminology. They must apply to, for example, morphogenesis and embryology. Rather than dismissing them as in the past as mere superstition, why not use them to inform the development of a new theory of morphogenesis, one based, for example, on the concept of a quantum morphogenetic field?

Let us take up the challenge presented by these and other mysterious physiological properties used in complementary medicine. The success of the originating disciplines both in diagnosis and in therapy constitutes a large body of empirical data. Nobel Laureate Sheldon Glashow once remarked that given any data, a theory could always be found- 'Give us the data, and we will find the theory'. The data presented by millennia of traditional CAM disciplines imply that fundamental explanatory theories for them must exist-as drug companies seeking new active ingredients from phytomedicine [or marine microorganisms (10)] well appreciate on a more practical level-there is some reason they work.

Here, a deeper perspective is being proposed, such data also constitute evidence in favour of the theories that inform them, as has been shown to be the case for Tridosha and the modus operandi of vibrational medicines. CAM disciplines can provide vital clues as to how to develop our understanding of biology itself. If scientists can rise to this challenge, CAM will stimulate the next paradigm shift in biology, one even deeper and more profound than present molecular biology resulting from elucidating the chemical structure of DNA.

Many years ago, based on considering the influence of electric fields on cell reproduction (43), Bentall had an inspired insight, leading him to conclude that 'Life is a harmonic' (R. Bentall, personal communication). Remarkably, quantized fluctuation fields describing the life force (18) are like harmonics of ordinary quantum fields. Similar, deeper insights surely await those who meet the challenge of CAM data and theories. Developing theories of CAM disciplines will produce great scientific advances, in addition to providing huge social benefits due to the increased scientific acceptability of, and therefore government backing for, CAM. Those who will benefit will not just be patients, but will include health professionals, scientists, institutions and governments as new, more effective treatments are accepted, and costs of maintaining the otherwise chronically ill are reduced.

\section{Acknowledgments}

I should like to thank B.D. Josephson PhD, T.S. Chang PhD, A. Scott-Morley BAc, R. Bentall MD, N. Clinch PhD and H. Pilcher MSc for conversations, T. Guinness, H. Pilcher,
S. Kurth and J. Alchin for financial support without which the research could not have been completed, and T. Darnell and The Prince of Wales Foundation for Integrative Health for helping stimulate its progress.

\section{References}

1. Astin JA. Why patients use alternative medicine: results of a national study. J Am Med Assoc 1998;279:1548-53.

2. Wootton JC. The White House commission on complementary and alternative medicine policy: meeting on the access to, and delivery of complementary and alternative services. J Altern Complement Med 2001;7:109-10.

3. Wootton JC, Sparber A. Surveys of complementary and alternative medicine: part I. General trends and demographic groups. J Altern Complement Med 2001;7:195-208.

4. Sparber A, Wootton JC. Surveys of complementary and alternative medicine: part II. Use of alternative and complementary cancer therapies. J Altern Complement Med 2001;7:281-8.

5. Wootton JC, Sparber A. Surveys of complementary and alternative medicine: part III. Use of alternative and complementary therapies in HIV/AIDS. J Altern Complement Med 2001;7:371-8.

6. Wootton JC, Sparber A. Surveys of complementary and alternative medicine: part IV. Use of alternative and complementary therapies for rheumatologic and other diseases. J Altern Complement Med 2001;7:715-24.

7. Wagner EH. Managing chronic disease. Br Med J 1999;318:1090.

8. Wagner EH. Chronic disease care. Br Med J 2004;328:177-8.

9. Wagner EH. Chronic disease management: what will it take to improve care for chronic illness? Effective Clin Pract 1998;1:2-4.

10. Cooper EL. Complementary and alternative medicine, when rigorous can be science. $e$ CAM 2004;1:1-8.

11. Hankey A. Daily Telegraph Letters 11 October 2004.

12. Jonas WB. The homeopathy debate. J Altern Complement Med 2000;6: 213-5.

13. House of Lords Select Committee on Science and Technology. Complementary and Alternative Medicine. 2000. Session 1999-2000 6th Report. The Stationary Office, London.

14. McIntyre MA. The House of Lords Select Committee's Report on CAM. J Altern Complement Med 2001;7:9-11.

15. Hankey A. Ayurvedic physiology and etiology: Ayurvedo Amritanam. The doshas and their functioning in terms of contemporary biology and physical chemistry. J Altern Complement Med 2001;7:388-96.

16. Hankey A. A test of the systems analysis underlying the theory of Ayurveda's Tridosha. J Altern Complement Med 2005; in press.

17. Hankey A. The scientific value of Ayurveda. J Altern Complement Med 2005; in press.

18. Hankey A. Are we close to a theory of energy medicine? J Altern Complement Med 2004;10:83-7.

19. Gerber R. Vibrational Medicine. Rochester, Vermont: Bear \& Co. 2001.

20. Hankey A. The principle that makes homeopathy scientifically possible: the whole is greater than the sum of its parts. Homeopathy 2004; in press.

21. Hankey A. Regulation biophysics in the nadi/acumeridian system. Proceedings of the IMEDIS Conference. Moscow, April 2004. Vol. I.

22. Scott-Morley A. Lecture Proceedings of the IMEDIS Conference. Moscow, April 2004. Vol. I.

23. Selye H. The Stress of Life. Maidenhead, UK: McGraw-Hill 1978.

24. Lee M. Regulators of G1 cyclin-dependent kinases and cancers. Cancer Metastasis Rev 2003;22:435-49.

25. Burns K. Agno J. Sicinski P. Matzuk M. Cyclin D2 and p27 are tissuespecific regulators of tumorigenesis in alpha knockout mice. Mol Endocrinol 2003;17:2053-69.

26. Fernandez P, Hernandez L, Farre X, Campo E, Cardesa A. Alteration of cell-cycle regulatory genes in prostate cancer. Pathobiology 2002; 70:1-10.

27. Hyland ME. Does complexity theory provide the basis for understanding the mechanisms underlying CAM. Handouts to integrated medicine conference 2001a. Royal College of Physicians, London.

28. Milgrom LR. Patient-practitioner-remedy (PPR) entanglement. Part 3. Refining the quantum metaphor for homeopathy. Homeopathy 2003;92: $152-60$.

29. Milgrom LR. Patient-practitioner-remedy (PPR) entanglement. Part 4. Towards classification and unification of different entanglement models for homeopathy. Homeopathy 2004;93:34-42. 
30. Milgrom LR. Patient-practitioner-remedy (PPR) entanglement. Part 7. A gyroscopic metaphor for the vital force and its use to model some of the empirical laws of homeopathy. Forsche Komp Klass Natur 2004; in press.

31. Milgrom LR. Patient-practitioner-remedy entanglement. Part 8. 'Laserlike' action of the homeopathic therapeutic encounter as predicted by a gyroscopic metaphor for the vital force. Forsch Komplementämed Klass Natur in press.

32. Torres J-L. Homeopathic effect: a network perspective. Homeopathy 2002;91;89-94.

33. Bodeker G. Evaluating Ayurveda. Guest Editorial. J Altern Complement Med 2001;7:389-92.

34. Frohlich H. Theoretical physics and biology. In: Frohlich H (ed.). Biological Coherence and Response to External Stimuli. Berlin: SpringerVerlag 1988, 1-24.

35. Smith CW. Quantum and coherence effects in water and living systems. J Altern Complement Med 2004;10:69-78.
36. Smith CW. Physicks and physics. J Altern Complement Med 1999;5: 191-3.

37. Sharma H, Clark C. Contemporary Ayurveda. Philadelphia: Churchill Livingstone 1998.

38. Sharma PV. Caraka Samhita. 4 Vols, 4th edn. Chowkambha Sanskrit Series Office, Varanasi, India: Chaukambha Orientalia 1981-1986.

39. Joshi R. A biostatistical approach to Ayurveda: quantifying the Tridosha. J Altern Comp Med 2004;10:879-89.

40. Patwardhan Bh. Joshi K. Chopra A. Classification of human population based on Ayurvedic concept of Prakriti. J Altern Complement. Med 2005; 11: in press.

41. Prigogine I, Stengers I. Order Out of Chaos. London, UK: Fontana 1985.

42. Reddy K. Conquering Chronic Disease Through Maharishi Vedic Medicine. Schenectady, NY: Samhita Productions 2002.

43. Bentall RHC. Electromagnetic energy: a historical therapeutic perspective: its future. In: O'Connor ME, Bentall RHC, Monahan JC (eds). Emerging Electromagnetic Medicine. New York, Springer-Verlag 1990. 


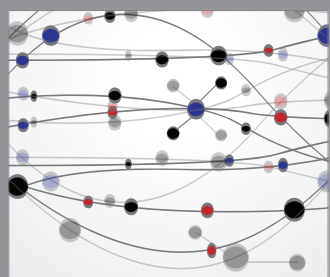

The Scientific World Journal
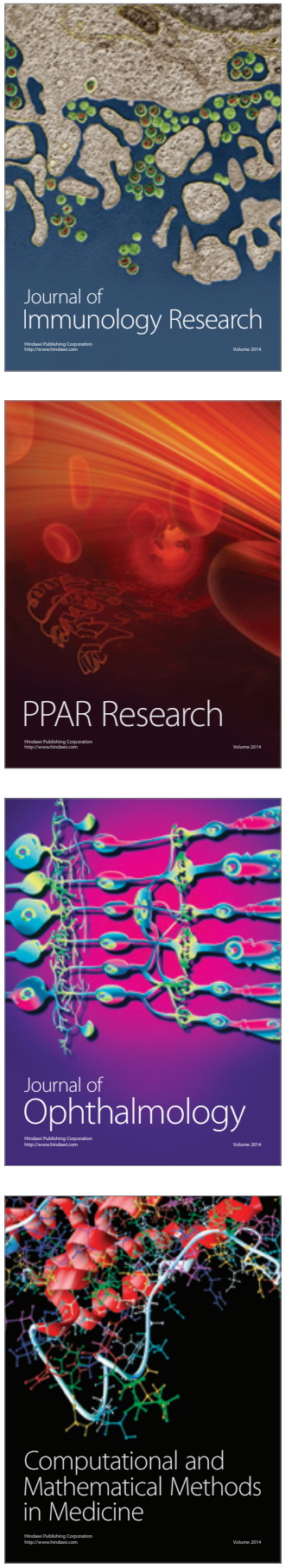

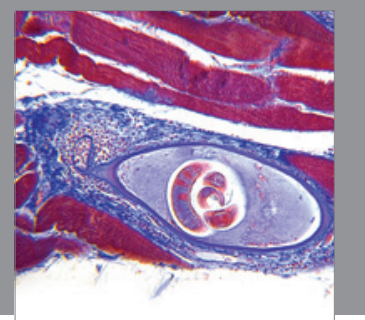

Gastroenterology

Research and Practice
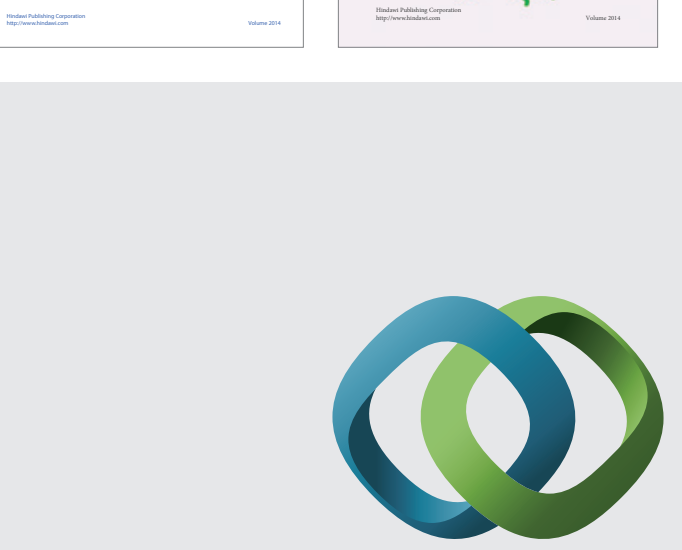

\section{Hindawi}

Submit your manuscripts at

http://www.hindawi.com
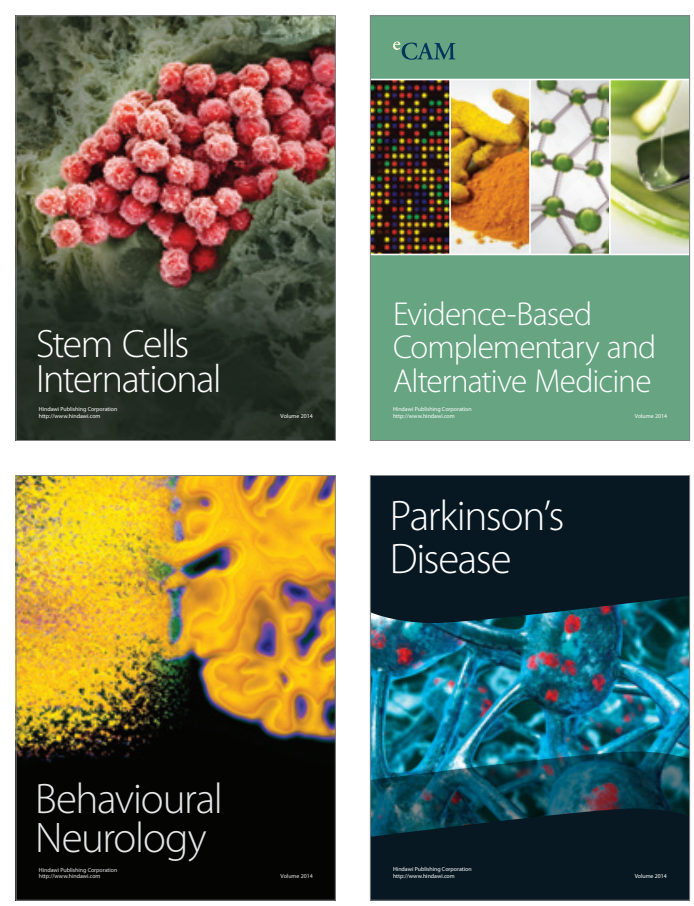

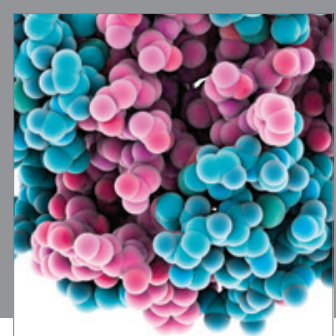

Journal of
Diabetes Research

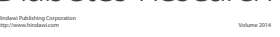

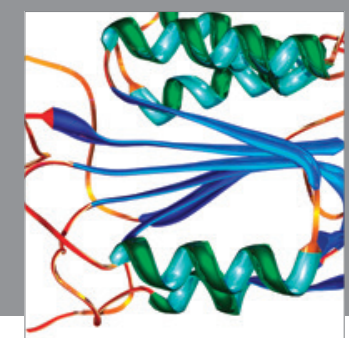

Disease Markers
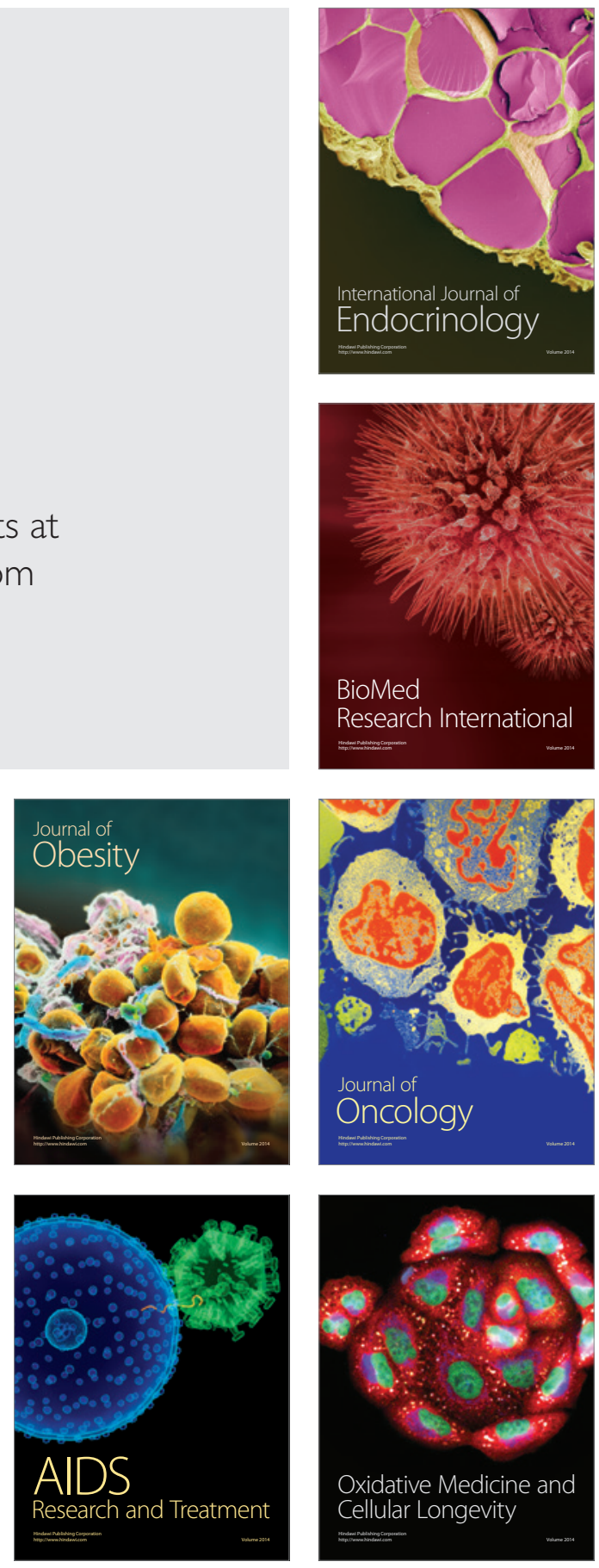